EPJ Web of Conferences 71, 00107 (2014)

DOI: 10.1051/epjconf/20147100107

(C) Owned by the authors, published by EDP Sciences, 2014

\title{
A Review of Recent Results on Quarkonium Production at LHCb
}

\author{
Monica Pepe Altarelli ${ }^{1}$,a \\ On behalf of the LHCb collaboration \\ ${ }^{1}$ CERN
}

\begin{abstract}
A selection of $\mathrm{LHCb}$ results is presented on the production of heavy quarkonium states in $p p$ collisions, including results on $J / \psi$ and $r$ production at $\sqrt{s}=8 \mathrm{TeV}$, the measurement of prompt $J / \psi$ polarisation, the production of $\chi_{c}$ mesons from converted photons, exclusive charmonium production, double $J / \psi$ production, as well as recent results on $J / \psi$ production in proton-lead collisions at $\sqrt{s_{\mathrm{NN}}}=5 \mathrm{TeV}$.
\end{abstract}

\section{Introduction}

Heavy quarkonium states are particularly interesting systems to test our understanding of strong interactions, both at perturbative and non-perturbative level, and are therefore the subject of intense theoretical and experimental studies. An effective field theory, non-relativistic QCD (NRQCD) [1, 2], provides the foundation for much of the current theoretical work. According to NRQCD, the production of heavy quarkonium factorises into two steps: a heavy quark-antiquark pair is first created at short distances and subsequently evolves non-perturbatively into quarkonium at long distances. The NRQCD calculations include colour-singlet (CS) and colour-octet (CO) amplitudes (see [3] and references therein), which account for the probability of a heavy quark-antiquark pair in a particular colour state to evolve into a heavy quarkonium state. The CS model (CSM) was initially used to describe experimental data. However, it underestimates the observed cross-section for single $J / \psi$ production at high transverse momentum $\left(p_{\mathrm{T}}\right)$. To resolve this discrepancy, the $\mathrm{CO}$ mechanism was introduced. More recent higher-order calculations to the CS predictions raise substantially the cross-sections at large $p_{\mathrm{T}}$ bringing them closer to the experimental data. However, none of these approaches can reproduce in a consistent way the available experimental results on both cross-section and polarisation [3].

Heavy charmonium is also produced from $b$-hadron decays. This production mechanism can be distinguished from prompt quarkonium production by exploiting the $b$-hadron finite decay time. In this case QCD predictions are based on the Fixed-Order-Next-to-Leading-Log (FONLL) formalism $[4,5]$, which combines a Fixed-Order calculation with an all-order resummation of Leading and Next-to-Leading logarithms.

Recent results from heavy quarkonium production at $\mathrm{LHCb}$ will be compared with various theoretical models to provide direct tests of the underlying production mechanism, including results on $J / \psi$ production in proton-lead collisions at $\sqrt{s_{\mathrm{NN}}}=5 \mathrm{TeV}$.

a e-mail: monica.pepe.altarelli@ cern.ch

This is an Open Access article distributed under the terms of the Creative Commons Attribution License 2.0, which permits unrestricted use, distribution, and reproduction in any medium, provided the original work is properly cited. 


\section{Production of $J / \psi$ and $\Upsilon(n S)$ mesons}

The differential production cross-sections of prompt $J / \psi$ and $\Upsilon(n S)$ (with $n=1,2,3$ ) mesons produced at the $p p$ collision point either directly or via feed-down from higher mass charmonium or bottomonium states, are measured at $\sqrt{s}=8 \mathrm{TeV}$ [6] with the LHCb detector [7]. The measurements are performed in the range of rapidity $2.0<y<4.5$ and $p_{\mathrm{T}}<14 \mathrm{GeV} / c$ for the $J / \psi$ meson and $p_{\mathrm{T}}<15 \mathrm{GeV} / c$ for the $\Upsilon(n S)$, in bins of $p_{\mathrm{T}}$ and $y$ with bin sizes $\Delta p_{\mathrm{T}}=1 \mathrm{GeV} / c$ and $\Delta y=0.5$. The fraction of $J / \psi$ from $b$-hadron decays (abbreviated as "from $b$ " in the following) is also measured in the same fiducial region. The $\Upsilon(n S)$ meson analysis is based on a data sample, corresponding to an integrated luminosity of about $51 \mathrm{pb}^{-1}$ of $p p$ interactions, while the analysis for the more abundant $J / \psi$ mesons is based on data, corresponding to an integrated luminosity of about $18 \mathrm{pb}^{-1}$. The $J / \psi$ and $\Upsilon(n S)$ mesons are reconstructed through their decay into a pair of muons. The analysis closely follows the strategy described in Refs. [8, 9] for $J / \psi$ production at $\sqrt{s}=7 \mathrm{TeV}$ and $2.76 \mathrm{TeV}$ and in [10] for $\Upsilon(n S)$ production at $\sqrt{s}=7 \mathrm{TeV}$. The resulting dimuon invariant mass distributions are displayed in Fig.1. The invariant mass resolution is on average $13.5 \mathrm{MeV} / \mathrm{c}^{2}$ for the $J / \psi$ and $43 \mathrm{MeV} / \mathrm{c}^{2}$ for the $\Upsilon(1 S)$ meson.

The production is studied under the assumption of zero polarisation, and no corresponding systematic uncertainty is assigned on the cross-section for this effect. This assumption is justified by the small polarisation measured by LHCb [11] for the $J / \psi$ meson at $\sqrt{s}=7 \mathrm{TeV}$, as reported in Sect.3, by ALICE [12] in a kinematic range similar to that used in this analysis, and by CMS [13] for the $\Upsilon(n S)$ at large $p_{\mathrm{T}}$ and central rapidity.

The measured differential cross-sections for the production of prompt $J / \psi$ mesons as a function of $p_{\mathrm{T}}$ are compared in Fig. 2 to three theoretical models: an NRQCD model at next-to-leading order (NLO) $[14,15]$, an NNLO* CSM [16, 17], where the notation NNLO* indicates that the calculation at next-to-next leading order is not complete and neglects part of the logarithmic terms and an NLO CSM [18]. In these comparisons the predictions are for direct $J / \psi$ meson production, i.e., they do not include feed-down from higher charmonium states, whereas the experimental measurements do include feed-down. In particular, the contribution from $J / \psi$ mesons produced in radiative $\chi_{\mathrm{c}}$ decays was measured by $\mathrm{LHCb}$ to be at the level of $20 \%$ at $\sqrt{s}=7 \mathrm{TeV}$ in the considered fiducial range[19]. Allowing for this contribution, both the NNLO* CSM and the NLO NRQCD models provide reasonable
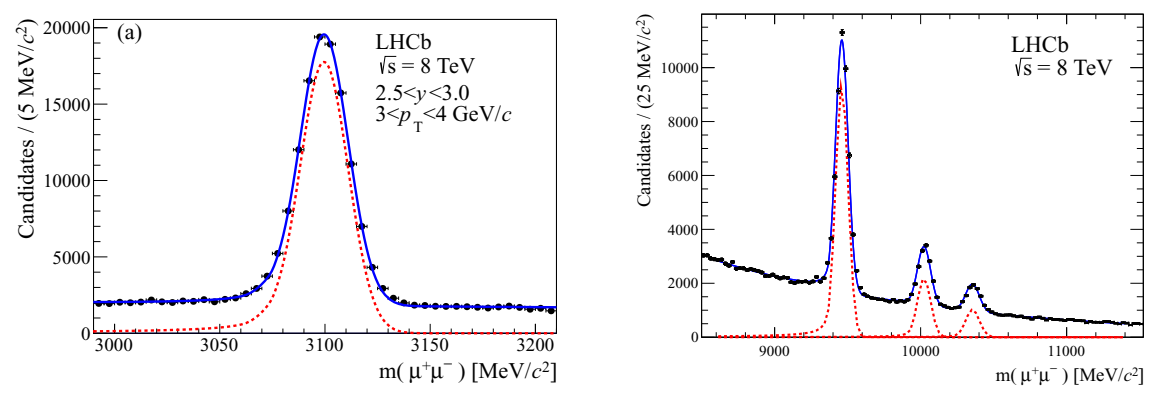

Figure 1. Dimuon invariant mass (left) of the selected $J / \psi \rightarrow \mu^{+} \mu^{-}$candidates in a selected bin in $y$ and $p_{\mathrm{T}}$ $\left(2.5<y<3.0\right.$ and $\left.3<p_{\mathrm{T}}<4 \mathrm{GeV} / c\right)$. Invariant mass distribution (right) of the selected $\Upsilon(n S) \rightarrow \mu^{+} \mu^{-}$ candidates in the range $p_{\mathrm{T}}<15 \mathrm{GeV} / c$ and $2.0<y<4.5$. The three peaks correspond to the $\Upsilon(1 S), \Upsilon(2 S)$ and $\Upsilon(3 S)$ meson signals (from left to right). 
descriptions of the experimental data. In contrast, the CSM at NLO underestimates the cross-section by an order of magnitude.

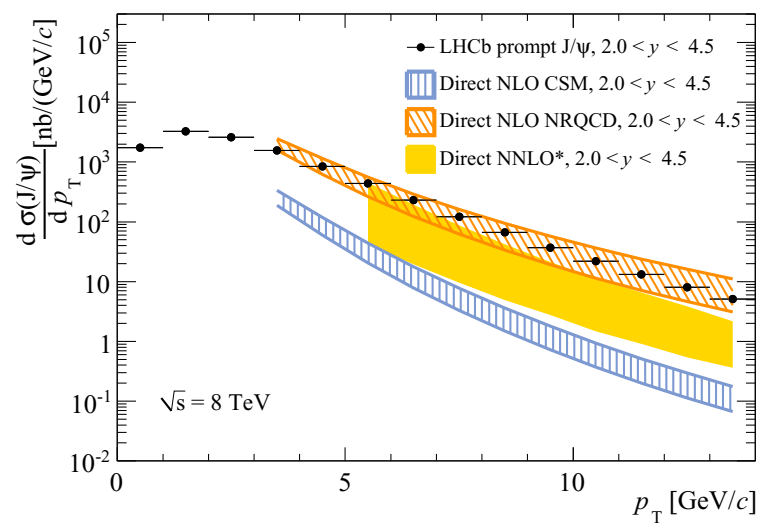

Figure 2. Comparison of the differential cross-section for the production of prompt $J / \psi$ meson (under the assumption of zero polarisation) as a function of $p_{\mathrm{T}}$ with direct production in an NLO NRQCD model $[14,15]$ (orange diagonal shading), an NNLO* CSM [17] (solid yellow) and an NLO CSM [18] (blue vertical shading).

The fraction of $J / \psi$ from $b$ is shown is Fig.3 as a function of the $J / \psi$ transverse momentum in bins of rapidity. This fraction is measured to be smaller at low $p_{\mathrm{T}}$ and for forward data. In Fig.4 (left) the data for the differential production cross-section for $J / \psi$ from $b$ as a function of $p_{\mathrm{T}}$ at $\sqrt{s}=8 \mathrm{TeV}$ are compared to the FONLL predictions. Very good agreement is observed. The cross-section for $J / \psi$ from $b$ production integrated over the defined fiducial region is determined to be $\sigma\left(J / \psi\right.$ from $\left.b, p_{\mathrm{T}}<14 \mathrm{GeV} / c, 2.0<y<4.5\right)=1.28 \pm 0.01 \pm 0.11 \mu \mathrm{b}$ [6], where the first uncertainty is statistical and the second is systematic, in very good agreement with the FONLL prediction of $1.34_{-0.49}^{+0.63} \mu \mathrm{b}[5]$.

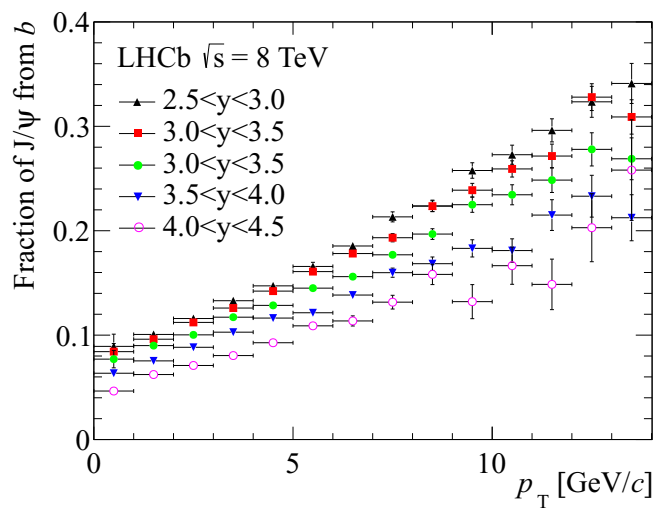

Figure 3. Fraction of $J / \psi$ from $b$ as a function of the $J / \psi$ transverse momentum, in bins of rapidity. 

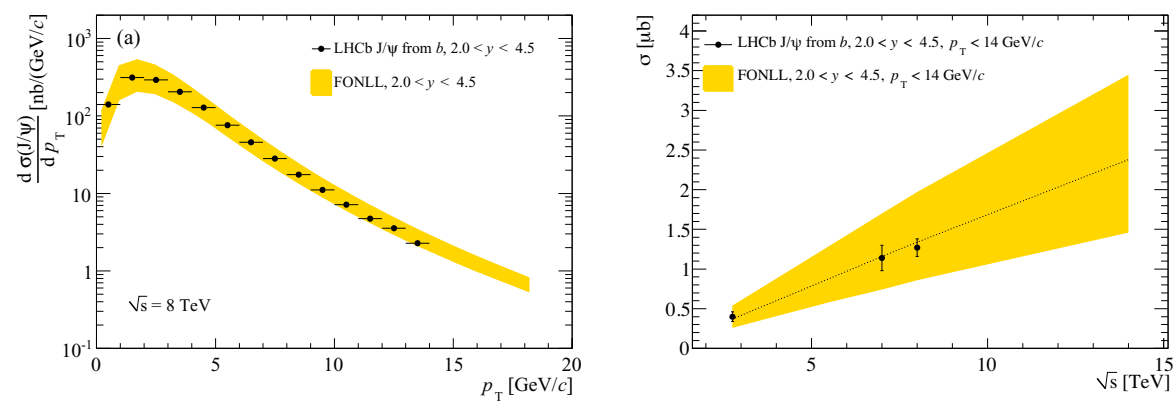

Figure 4. Differential production cross-section for $J / \psi$ from $b$ as a function of $p_{\mathrm{T}}$ in the fiducial range $2.0<y<$ 4.5 (left). The FONLL prediction $[4,5]$ is shown in yellow. Predictions based on the FONLL formalism $[4,5]$ for the production cross-section for $J / \psi$ from $b$ as a function of $\sqrt{s}$ (right) in the fiducial range $0<p_{\mathrm{T}}<14 \mathrm{GeV} / c$ and $2.0<y<4.5$. The uncertainty includes contributions from the renormalisation scale, quark masses and the choice of PDF set. The black dotted line shows the central value of the prediction. The points show the LHCb measurements at $\sqrt{s}=2.76$ [9], 7 [8], and $8 \mathrm{TeV}[6]$.

The results on $J / \psi$ production at $\sqrt{s}=8 \mathrm{TeV}$ provide an extra measurement, which complements those already published by LHCb at $\sqrt{s}=2.76$ [9] and $7 \mathrm{TeV}$ [8]. Figure 4 (right) shows the results for the cross-section for $J / \psi$ from $b$ at $\sqrt{s}=2.76,7$ and $8 \mathrm{TeV}$ as well as the FONLL prediction as a function of centre-of-mass energy. Excellent agreement with the theoretical calculation is observed.

From the measurement of the production cross-section for $J / \psi$ from $b$ in the fiducial region, using the LHCb Monte Carlo simulation based on Pүтніа to extrapolate to the full polar angle range, with an extrapolation factor of 5.4, and the inclusive $b \rightarrow J / \psi X$ branching fraction, one can derive the total $b \bar{b}$ production cross-section at $\sqrt{s}=8 \mathrm{TeV}, \sigma(p p \rightarrow b \bar{b} X)=298 \pm 2 \pm 36 \mu \mathrm{b}$, where the first uncertainty is statistical and the second is systematic.

In Fig. 5 the cross-sections times dimuon branching fractions for the three $\Upsilon$ meson states are compared to an NNLO* CSM [16] and an NLO CSM [18] as a function of $p_{\mathrm{T}}$. The NNLO* CSM provides a reasonable description of the experimental data, particularly for the $\Upsilon(3 S)$ meson, which is expected to be little affected by feed-down. As in the case of prompt $J / \psi$ meson production, the CSM at NLO underestimates the cross-section by an order of magnitude.
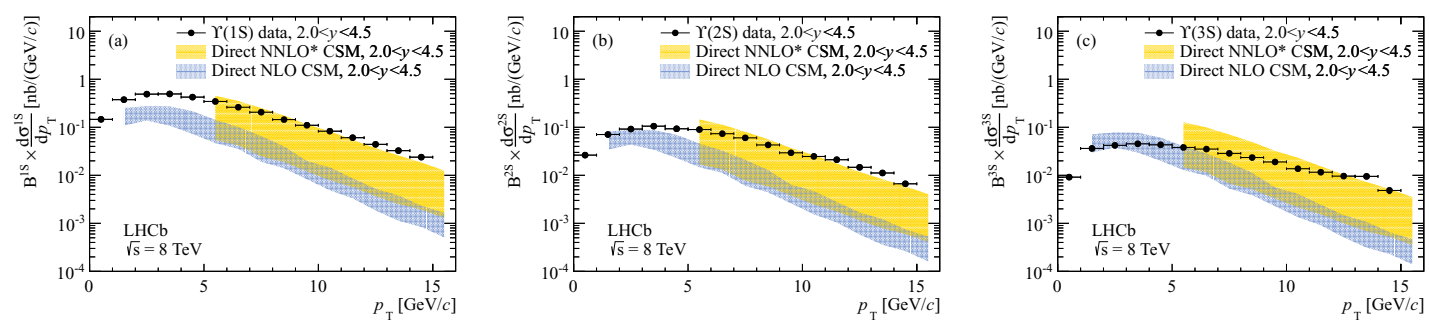

Figure 5. Comparison of the differential production cross-sections times dimuon branching fractions for (a) $\Upsilon(1 S)$, (b) $\Upsilon(2 S)$ and (c) $\Upsilon(3 S)$ mesons as a function of $p_{\mathrm{T}}$ with direct production in an NNLO* CSM [16] (solid yellow) and an NLO CSM [18] (blue vertical shading) model. 


\section{Polarisation of $J / \psi$ mesons}

Predicting the measured dependence of $J / \psi$ polarisation on $p_{\mathrm{T}}$ is one of the most difficult challenges for models of quarkonium production. In fact, even those models that succeed in describing reasonably well the magnitude and shape of the cross-section as a function of $p_{\mathrm{T}}$, such as NLO NRQCD, fail to describe polarisation data.

LHCb has performed an angular analysis of the $J / \psi$ decay into two muons to measure the polarisation of prompt $J / \psi$ mesons [11]. The analysis is based on a dataset corresponding to an integrated luminosity of $37 \mathrm{pb}^{-1}$ of $p p$ interactions at $7 \mathrm{TeV}$. The kinematic range covered by the measurement is $2<p_{\mathrm{T}}<15 \mathrm{GeV} / c$ and $2.0<y<4.5$. The angular distribution of the $J / \psi$ decay products can be expressed in its general form as

$$
\frac{d^{2} N}{d \cos \theta \mathrm{d} \phi} \propto 1+\lambda_{\theta} \cos ^{2} \theta+\lambda_{\theta \phi} \sin 2 \theta \cos \phi+\lambda_{\phi}, \sin ^{2} \theta \cos 2 \phi,
$$

in which $\theta$ is the polar angle of the positively-charged muon with respect to the $J / \psi$ polarisation axis and $\phi$ is the azimuthal angle with respect to the production plane formed by the momenta of the colliding protons in the $J / \psi$ rest frame. The analysis is performed in the helicity frame, in which the $J / \psi$ polarisation axis coincides with the $J / \psi$ flight direction in the centre-of-mass-frame of the colliding protons, and was cross-checked in the Collins-Soper frame [20]. The three parameters $\lambda_{\theta}$, $\lambda_{\theta \phi}$ and $\lambda_{\phi}$ quantify the degree of polarisation. When $\lambda_{\phi}=0$ and $\lambda_{\theta \phi}=0$, the values $\lambda_{\theta}=+1,-1,0$ correspond to fully transverse, fully longitudinal, and no polarisation, respectively. The measurement is performed by fitting the efficiency-corrected angular distribution of the data. The reliability of the simulation used to determine the efficiency is checked by studying a control channel, $B^{+} \rightarrow J / \psi K^{+}$, in which the $J / \psi$ is known to be longitudinally polarised in the $B^{+}$rest-frame.

The polarisation parameters $\lambda_{\phi}$ and $\lambda_{\theta \phi}$ are measured to be consistent with zero, within uncertainties. The parameter $\lambda_{\theta}$ shows a small longitudinal polarisation, as displayed in Fig.6 (left) as a function of $p_{\mathrm{T}}$ in five rapidity intervals in the helicity frame. The average of $\lambda_{\theta}$ over all $\left(p_{\mathrm{T}}, y\right)$ bins, weighted according to the number of events in each bin, is $-0.145 \pm 0.027$. Similar results are obtained in the Collins-Soper frame. Figure 6 (right) shows a comparison with the results obtained by ALICE [21] for inclusive $J / \psi$ production at $7 \mathrm{TeV}$ in a kinematic region similar to that of $\mathrm{LHCb}$ $\left(2<p_{\mathrm{T}}<8 \mathrm{GeV} / c\right.$ and $\left.2.5<y<4.0\right)$. The comparison, which is performed in the common rapidity interval $2.5<y<4$.0, shows good agreement. The CMS collaboration has also published [22] a mea-
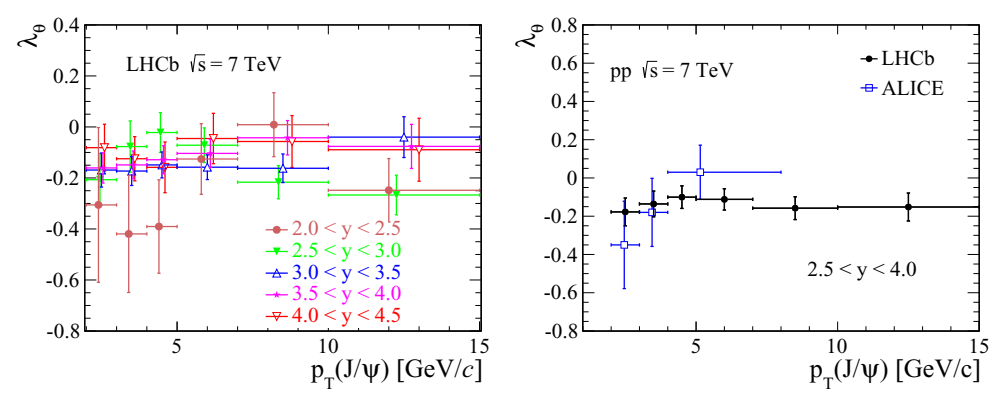

Figure 6. Measurements of $\lambda_{\theta}$ in bins of $p_{\mathrm{T}}$ for five rapidity bins (left) in the helicity frame. Comparison of $\mathrm{LHCb}$ and ALICE results for $\lambda_{\theta}$ in bins of $p_{\mathrm{T}}$ integrating over $2.5<y<4.0$ (right) in the helicity frame. 


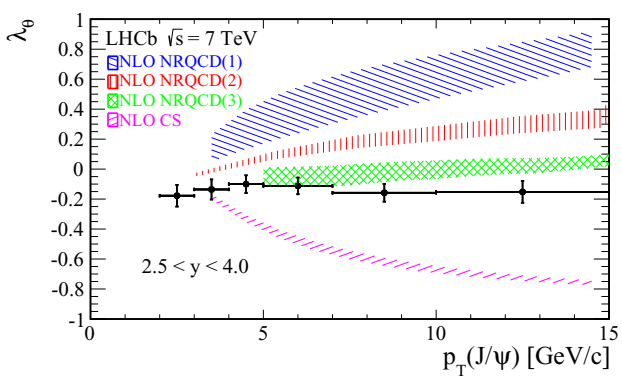

Figure 7. Comparison of LHCb results for $\lambda_{\theta}$ as a function of $p_{\mathrm{T}}$ with an NLO color singlet model [23] and three different NLO NRQCD calculations [23-25] in the helicity frame. These theoretical predictions do not include feed-down from excited states, which are however predicted to be rather small.

surement of the prompt $J / \psi$ polarisation for $14<p_{\mathrm{T}}<70 \mathrm{GeV} / c$ in the rapidity intervals $|y|<0.6$ and $0.6<|y|<1.2$ without finding any evidence of large transverse or longitudinal polarisation.

Figure 7 compares the LHCb results for $\lambda_{\theta}$ as a function of $p_{\mathrm{T}}$ with an NLO color singlet model [23] and three different NLO NRQCD calculations [23-25] in the helicity frame. The NLO color singlet model predicts a strong longitudinal polarisation of the $J / \psi$ meson and does not describe the data. Predictions based on NLO NRQCD vary dramatically depending on the data used to determine the NRQCD matrix elements. A prediction of near-zero transverse polarisation only arises for those fits of the NRQCD matrix elements that include both data on hadro-production and the CDF Run II polarisation measurements [26].

\section{Production of $\chi_{\mathrm{c} 0}, \chi_{\mathrm{c} 1}$ and $\chi_{\mathrm{c} 2}$ mesons}

Prompt production of $\chi_{\mathrm{c} 0}, \chi_{\mathrm{c} 1}$ and $\chi_{\mathrm{c} 2}$ mesons is studied by LHCb using an integrated luminosity of $1 \mathrm{fb}^{-1}$ of $p p$ interactions at $7 \mathrm{TeV}$ [27]. The ratio of the prompt $\chi_{\mathrm{c} 2}$ to $\chi_{\mathrm{c} 1}$ production cross-sections is measured as a function of the $J / \psi$ transverse momentum from 3 to $30 \mathrm{GeV} / c$, in the rapidity range $2.0<y<4.5$. The $\chi_{c}$ mesons are identified through their decay $\chi_{c} \rightarrow J / \psi \gamma$, where the $J / \psi$ is reconstructed in the dimuon final state and photons are required to convert in the detector material before the magnet, as sketched in Fig. 8 (left). Converted photons, i.e., photons reconstructed from
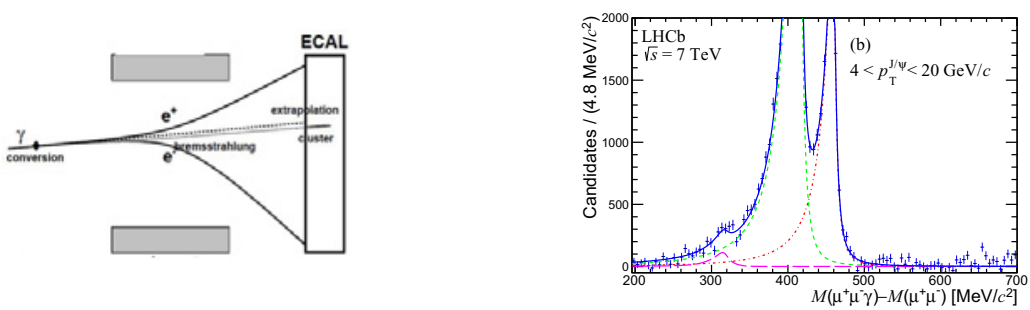

Figure 8. Sketch illustrating the conversion of a photon before the LHCb dipole magnet (left) and the bremsstrahlung correction to the charged electron candidates. Distribution of $\Delta M=M\left(\mu^{+} \mu^{-} \gamma\right)-M\left(\mu^{+} \mu^{-}\right)$ (right) for $4<p_{\mathrm{T}}^{J / \psi}<20 \mathrm{GeV} / c$ after background subtraction. The $\chi_{\mathrm{c} 0}, \chi_{\mathrm{c} 2}$ and $\chi_{\mathrm{c} 2}$ signals are clearly visible. 
a pair of oppositely charged electron candidates, are in fact characterised by an energy resolution that is better than that of calorimetric photons. As a result, the $\chi_{\mathrm{c} 1}$ and $\chi_{\mathrm{c} 2}$ peaks can be nicely resolved, however at the prize of a reduced efficiency. The ratio of efficiencies of converted photons to calorimetric photons is measured from data to be only around $2 \%$ using a sample of $\pi^{0}$ mesons, reconstructed either with two calorimetric photons or with one calorimetric and one converted photon. A bremsstrahlung correction is applied to each electron track by adding the energy of photons in the electromagnetic calorimeter (ECAL), whose position is compatible with a straight line extrapolation of the electron track. Figure 8 (right) shows the distribution of $\Delta M=M\left(\mu^{+} \mu^{-} \gamma\right)-M\left(\mu^{+} \mu^{-}\right)$for $4<p_{\mathrm{T}}^{\mathrm{J} / \psi}<20 \mathrm{GeV} / c$ after background subtraction. In addition to the $\chi_{\mathrm{c} 1}$ and $\chi_{\mathrm{c} 2}$ signals, a $\chi_{\mathrm{c} 0}$ signal is observed for the first time at a hadron collider with a statistical significance of $4.3 \sigma$.

Figure 9 shows the ratio of the $\chi_{\mathrm{c} 2}$ to $\chi_{\mathrm{c} 1}$ production cross-sections as a function of $p_{\mathrm{T}}^{J / \psi}$, under the assumption that the $\chi_{c}$ mesons are produced unpolarised. The measurements are compared with NLO NRQCD [28] and LO NRQCD [29] calculations. For relatively large $p_{\mathrm{T}}$ the theoretical calculations are in reasonable agreement with the data points.

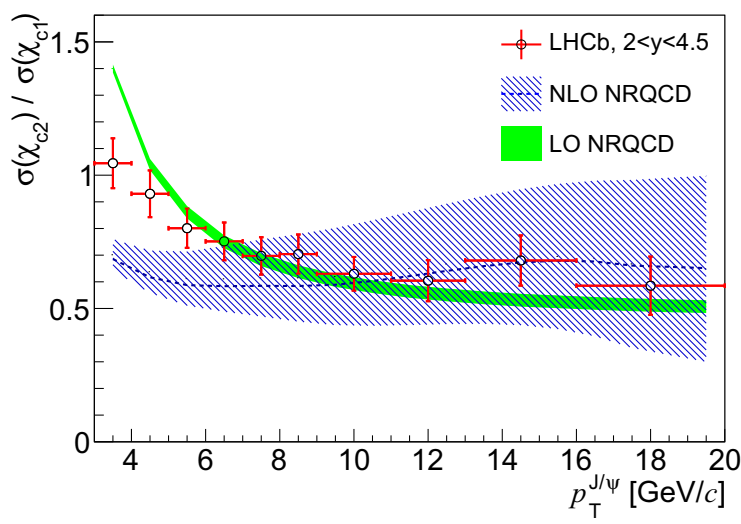

Figure 9. Comparison of the $\mathrm{LHCb}$ results on the ratio of the $\chi_{\mathrm{c} 2}$ to $\chi_{\mathrm{c} 2}$ production cross-sections as a function of $p_{\mathrm{T}}^{J / \psi}$ with NLO NRQCD [28] and LO NRQCD [29] calculations.

\section{Double $J / \psi$ production}

Given the large charmonium production cross-section at the LHC, the question of multiple production naturally arises. This was studied by LHCb using an integrated luminosity of about $37 \mathrm{pb}^{-1}$ of $p p$ interactions at $7 \mathrm{TeV}$ [30]. Simultaneous production of two $J / \psi$ mesons can be expected in single parton collisions via higher order $O\left(\alpha_{s}^{4}\right)$ gluon-gluon diagrams. This production could also be enhanced by double parton scattering (DPS) in which two gluons are extracted from a proton and the two $J / \psi$ mesons are produced in two independent sub-processes. The cross-section for double $J / \psi$ production in the fiducial range $2<y^{J / \psi}<4.5$ and $p_{\mathrm{T}}^{J / \psi}<10 \mathrm{GeV} / c$ is measured to be $\sigma^{J / \psi J / \psi}=5.1 \pm 1.0 \pm 1.1 \mathrm{nb}[30]$, where the first uncertainty is statistical and the second is systematic, in good agreement with predictions from leading-order QCD calculations [31]. The suppression factor with respect to single prompt $J / \psi$ production is $\sigma^{J / \psi J / \psi} / \sigma^{J / \psi}=\left(5.1 \pm 1.0 \pm 0.6_{-1.0}^{+1.2}\right) \times 10^{-4}$, where the first uncertainty is statistical, the second systematic and the third accounts for the unknown $J / \psi$ polarisation. Figure 10 shows the differential production cross-section for $J / \psi$ pairs as a function of 


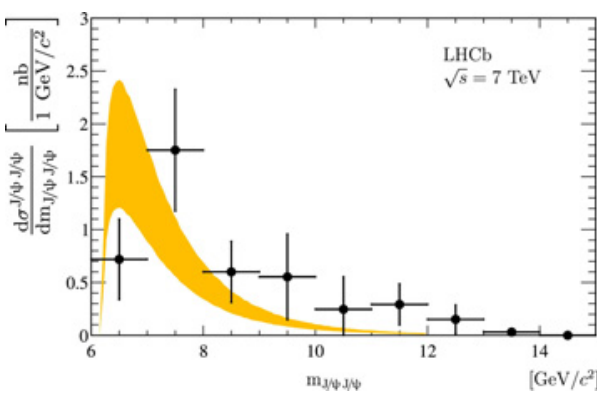

Figure 10. Differential production cross-section for $J / \psi$ pairs as a function of the invariant mass of the $J / \psi$ pair system. The shaded area corresponds to the prediction by the model described in Ref. [31].

the invariant mass of the $J / \psi$ pair system. The data are compared with a theoretical prediction [31], which includes both direct production and feed-down from $\psi(2 S)$ decays and no contribution from DPS. Within the available statistics good agreement is observed. For double $J / \psi$ production, the predictions from single and double parton scattering are expected to be fairly close in magnitude [32], but with somewhat different kinematic distributions for the $J / \psi$ pairs. The analysis of the higher statistics already collected by $\mathrm{LHCb}$ will allow an in-depth study of the kinematic properties of double $J / \psi$ production and of the different production models. For completeness, it should also be mentioned that LHCb has already published an analysis of the production of $J / \psi$ mesons accompanied by open charm, and of pairs of open charm hadrons based on a sample of $355 \mathrm{pb}^{-1}$ of $p p$ interactions at $7 \mathrm{TeV}[33]$.

\section{Exclusive production of $J / \psi$ and $\psi(2 S)$ mesons}

Exclusive production of $J / \psi$ and $\psi(2 S)$ mesons through processes such as the one displayed in Fig.11 were observed by LHCb [34] in the dimuon channel. LHCb is very well suited for this type of studies as it has access to high rapidities, with some sensitivity to backward tracks using the Vertex Locator (VELO), it operates at relatively low pileup and has sensitivity to low momentum and transverse momentum particles. Exclusively produced $J / \psi$ and $\psi(2 S)$ mesons into dimuons are characterised by a very distinct topology: their selection requires the presence of two reconstructed muons in the forward region and no other tracks and photons in the detector. An additional rapidity gap is obtained by also excluding tracks in the backward region using the VELO detector. Based on a data sample, corresponding to an integrated luminosity of about $36 \mathrm{pb}^{-1}$ of $p p$ interactions, the cross-sections times

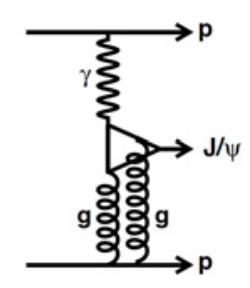

Figure 11. Feynman diagram contributing to exclusive $J / \psi$ photoproduction. 
branching fractions to two muons for exclusive $J / \psi$ and $\psi(2 S)$ with pseudorapidities between 2.0 and 4.5 is measured to be $\sigma_{p p \rightarrow J / \psi\left(\rightarrow \mu^{+} \mu^{-}\right)}=307 \pm 21 \pm 36 \mathrm{pb}$ and $\sigma_{p p \rightarrow \psi(2 S)\left(\rightarrow \mu^{+} \mu^{-}\right)}=7.8 \pm 1.3 \pm 1.0 \mathrm{pb}$, where the first uncertainty is statistical and the second is systematic, in good agreement with theoretical predictions [35-38]. During the 2012 run, a new trigger was implemented to increase the rate of exclusive events, which makes use of "upstream" silicon sensors to veto any backward activity and of soft $p_{\mathrm{T}}$ cuts to gain sensitivity for hadronic final states. Data corresponding to an integrated luminosity of about $1.4 \mathrm{fb}^{-1}$ were already collected with such a trigger, which will allow LHCb to study the production of charmonium decaying into hadronic final states, the production of open charm and to perform a search for higher mass charmonium states.

\section{Production of $J / \psi$ mesons in proton-lead collisions at $\sqrt{s_{\mathrm{NN}}}=5 \mathrm{TeV}$}

$\mathrm{LHCb}$ has recently published results on production of $\mathrm{J} / \psi$ mesons in proton-lead collisions at $\sqrt{s_{\mathrm{NN}}}=5 \mathrm{TeV}$ [39]. These studies are important as they allow disentangling the effects of quarkgluon plasma from so-called cold nuclear matter effects (see, e.g., [40-42]). Data were collected with a proton beam energy of $4 \mathrm{TeV}$ and a lead beam energy of $1.58 \mathrm{TeV}$ per nucleon, resulting in a centreof-mass energy of the proton-nucleon system of $5 \mathrm{TeV}$. The instantaneous luminosity was around $510^{27} \mathrm{~cm}^{-2} \mathrm{sec}^{-1}$, i.e., five orders of magnitude below the typical LHCb luminosity for $p p$ collisions. The directions of the proton and lead beams were swapped during data taking to produce both $p A$ and $A p$ collisions. Data collected with the inverted beam directions (i.e., in Ap collisions) allow LHCb to measure backward production. The proton-nucleon centre-of-mass system has a rapidity in the laboratory frame of $+0.47(-0.47)$ for $p A(A p)$ collisions, resulting in a rapidity coverage in the laboratory frame ranging from about 1.5 to $4.0(-5.0$ to -2.5$)$ for the $p A(A p)$ samples. The analysis is based on data sets corresponding to integrated luminosities of $1.1 \mathrm{nb}^{-1}$ of $p A$ and $0.5 \mathrm{nb}^{-1}$ of $A p$ collisions. The single-differential production cross-sections as functions of $y$ and $p_{\mathrm{T}}$ are measured for prompt $J / \psi$ and $J / \psi$ from $b$ for $p A$ and $A p$ collisions. From the cross-sections one can derive the nuclear modification factor, a quantity that is normally is used to characterise nuclear effects. The nuclear modification factor is defined as the production cross-section of a given particle per nucleon in $p$ A collisions divided by that in $p p$ collisions: $R_{p A}\left(y, \sqrt{s_{\mathrm{NN}}}\right)=\frac{1}{A} \frac{\mathrm{d} \sigma_{p A}\left(y, \sqrt{s_{\mathrm{NN}}}\right) / \mathrm{d} y}{\mathrm{~d} \sigma_{p p}\left(y, \sqrt{s_{\mathrm{NN}}}\right) / \mathrm{d} y}$. A power-law interpolation of the published measurements at $\sqrt{s}=2.76$ [9], 7 [8], and $8 \mathrm{TeV}$ [6] is used to compute the reference $J / \psi$ production cross-section in $p p$ collisions at $5 \mathrm{TeV}$. Figure 12 shows the results for the nuclear modification factor calculated in the rapidity ranges $-4.0<y<-2.5$ and $2.5<y<4.0$, separately for (left) prompt $J / \psi$ and (right) $J / \psi$ from $b$ and compares them to various theoretical predictions [40-42]. A suppression of about $40 \%$ at large rapidity is observed for prompt $J / \psi$ production, in reasonable agreement with most predictions. For $J / \psi$ from $b$ the suppression is more modest and, again, in good agreement with the theory.

\section{Conclusion}

LHCb has performed a wealth of measurements on quarkonium production, and many more are coming! A few selected ones were briefly illustrated in this report.

Measurements of charmonium [6, 8, 9] and bottomonium [6, 10] production were performed at various centre-of-mass energies. They allow an in depth comparison with theoretical models. A simple CSM is disfavoured by the data, while a combination of CS and CO, as implemented in the NRQCD formalism, or CS improved by QCD corrections, provide a good description of prompt quarkonium production. These models, however, do not succeed in reproducing the data on polarisation [11]. Charmonium production from $b$-hadron decays is very well described by FONLL 

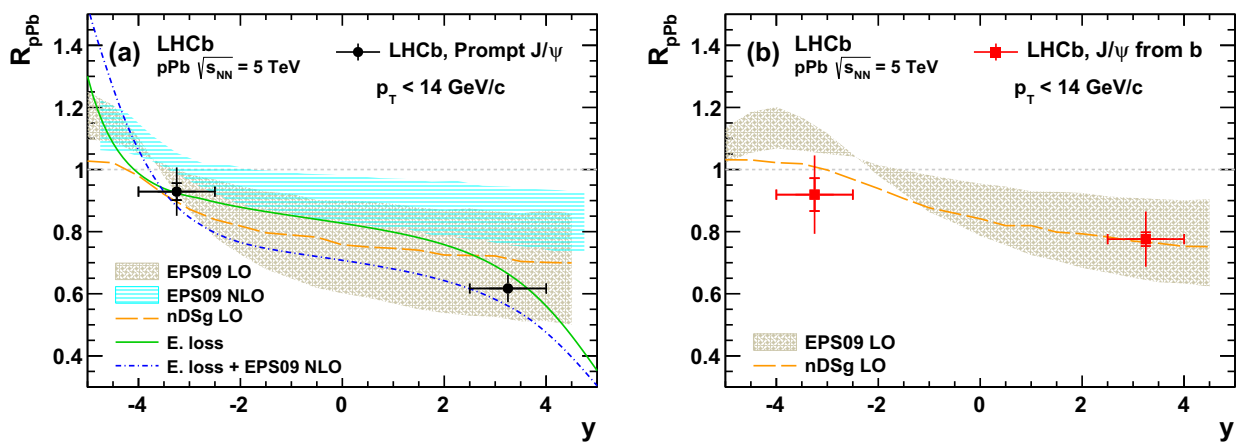

Figure 12. Nuclear modification factor in proton-lead collisions at $\sqrt{s_{\mathrm{NN}}}=5 \mathrm{TeV}$ as a function of $y$ for (left) prompt $J / \psi$ mesons and (right) $J / \psi$ from $b$. The results are compared to theoretical predictions from (yellow dashed line and brown band) [40], (blue band) [41] and (green solid and blue dash-dotted lines) [42].

calculations. Some other results were illustrated in this report, such as the production of $\chi_{c}$ mesons from converted photons with the first observation of a $\chi_{\mathrm{c} 0}$ signal at a hadron collider [27], exclusive charmonium production [34], double $J / \psi$ production [30], as well as results on $J / \psi$ production in proton-lead collisions at $\sqrt{s_{\mathrm{NN}}}=5 \mathrm{TeV}$ [39]. Some of these results are based on the analysis of a partial dataset and will gain in precision with the analysis of the full data sample available to LHCb.

\section{Acknowledgments}

I would like to warmly thank the Organisers of ICNFP 2013, Larissa Bravina, Yiota Foka and Sonia Kabana, for their kind invitation and their beautiful hospitality in such a great setting. I would also like to thank my LHCb colleagues for providing the material discussed here and, in particular, Giulia Manca and Vanya Belyaev for their careful reading of this article.

\section{References}

[1] W.E. Caswell, G.P. Lepage, Phys.Lett. B167, 437 (1986)

[2] G.T. Bodwin, E. Braaten, G.P. Lepage, Phys. Rev. D 51, 1125 (1995)

[3] N. Brambilla et al., Eur. Phys. J. C71, 1534 (2011), 1010. 5827

[4] M. Cacciari, M. Greco, P. Nason, JHEP 05, 007 (1998), hep-ph/9803400

[5] M. Cacciari, S. Frixione, N. Houdeau, M.L. Mangano, P. Nason et al., JHEP 10, 137 (12), 1205.6344

[6] R. Aaij et al. (LHCb collaboration), JHEP 1306, 064 (2013), 1304. 6977

[7] A.A. Alves Jr. et al. (LHCb collaboration), JINST 3, S08005 (2008)

[8] R. Aaij et al. (LHCb collaboration), Eur. Phys. J. C71, 1645 (2011), 1103.0423

[9] R. Aaij et al. (LHCb collaboration), JHEP 02, 041 (2013), 1212.1045

[10] R. Aaij et al. (LHCb collaboration), Eur.Phys.J. C72, 2025 (2012), 1202.6579

[11] R. Aaij et al. (LHCb collaboration) (2013), to appear in Eur. Phys. J. C, 1307.6379

[12] B. Abelev et al. (ALICE collaboration), Phys.Rev.Lett. 108, 082001 (2012), 1111. 1630

[13] S. Chatrchyan et al. (CMS collaboration), Phys.Rev.Lett. (2012), 1209.2922 
[14] M. Butenschön, B.A. Kniehl, Phys.Rev. D84, 051501 (2011), 1105.0820

[15] M. Butenschön, B.A. Kniehl, Phys.Rev.Lett. 106, 022003 (2011), 1009.5662

[16] P. Artoisenet, J.M. Campbell, J. Lansberg, F. Maltoni, F. Tramontano, Phys.Rev.Lett. 101, 152001 (2008), 0806. 3282

[17] J.P. Lansberg, Eur. Phy. J. C 61, 693 (2008), 0811 . 4005

[18] J.M. Campbell, F. Maltoni, F. Tramontano, Phys.Rev.Lett. 98, 252002 (2007), hep-ph/0703113

[19] R. Aaij et al. (LHCb collaboration), Phys.Lett. B718, 431 (2012), 1204.1462

[20] J.C. Collins, D.E. Soper, Phys. Rev. D 16, 2219 (1977)

[21] B. Abelev et al. (ALICE collaboration), Phys.Rev.Lett. 108, 082001 (2012), 1111.1630

[22] S. Chatrchyan et al. (CMS collaboration) (2013), 1307.6070

[23] M. Butenschoen, B.A. Kniehl, Nucl.Phys.Proc.Suppl. 222-224, 151 (2012), 1201.3862

[24] B. Gong, L.P. Wan, J.X. Wang, H.F. Zhang, Phys.Rev.Lett. 110, 042002 (2013), 1205.6682

[25] K.T. Chao, Y.Q. Ma, H.S. Shao, K. Wang, Y.J. Zhang, Phys.Rev.Lett. 108, 242004 (2012), 1201.2675

[26] A. Abulencia et al. (CDF collaboration), Phys.Rev.Lett. 99, 132001 (2007), 0704.0638

[27] R. Aaij et al. (LHCb collaboration) (2013), to appear in JHEP, 1307.4285

[28] Y.Q. Ma, K. Wang, K.T. Chao, Phys.Rev. D83, 111503 (2011), 1002 . 3987

[29] A. Likhoded, A. Luchinsky, S. Poslavsky (2013), 1305. 2389

[30] R. Aaij et al. (LHCb collaboration), Phys.Lett. B707, 52 (2012), 1109. 0963

[31] A. Berezhnoy, A. Likhoded, A. Luchinsky, A. Novoselov, Phys.Rev. D84, 094023 (2011), 1101.5881

[32] A.V. Berezhnoy, A.K. Likhoded, A.V. Luchinsky, A.A. Novoselov, Phys. Rev. D 86, 034017 (2012)

[33] R. Aaij et al. (LHCb collaboration), JHEP 06, 141 (2012), 1205. 0975

[34] R. Aaij et al. (LHCb collaboration), J.Phys. G40, 045001 (2013), 1301.7084

[35] L. Harland-Lang, V. Khoze, M. Ryskin, W. Stirling, Eur.Phys.J. C65, 433 (2010), 0909. 4748

[36] S.R. Klein, J. Nystrand, Phys. Rev. Lett. 92, 142003 (2004)

[37] L. Motyka, G. Watt, Phys.Rev. D78, 014023 (2008), 0805. 2113

[38] V. Goncalves, M. Machado, Phys.Rev. C84, 011902 (2011), 1106. 3036

[39] R. Aaij et al. (LHCb collaboration) (2013), submitted to JHEP, 1308.6729

[40] E. Ferreiro, F. Fleuret, J. Lansberg, A. Rakotozafindrabe, Phys.Rev. C88, 047901 (2013), 1305.4569

[41] J. Albacete, N. Armesto, R. Baier, G. Barnafoldi, J. Barrette et al., Int.J.Mod.Phys. E22, 1330007 (2013), 1301.3395

[42] F. Arleo, S. Peigne, JHEP 1303, 122 (2013), 1212 . 0434 